\title{
educação

\section{Atuar na indução de professores: Que implicações para os diretores escolares portugueses?}

\author{
Marta Mateus Almeidai, Estela Costa ${ }^{\mathrm{II}}$, Ana Sofia \\ PINHO ${ }^{\mathrm{III}}$, JOANA PIPA ${ }^{\mathrm{IV}}$ \\ Universidade de Lisboa, Portugal
}

O artigo centra-se no papel desempenhado pelos diretores escolares portugueses na promoção da integração e do desenvolvimento profissional dos professores principiantes nas suas organizações. Desenvolvido no quadro do Projeto Europeu The Outstanding New Teacher Programme (ONTP), o artigo apresenta os resultados do estudo de cariz quantitativo, com recurso à técnica de questionário, aplicado em formato eletrónico, procurando-se perceber que necessidades são sentidas por estes diretores para acolherem e potenciarem o crescimento profissional dos professores em início de atividade. Os dados recolhidos foram analisados recorrendo a dois procedimentos: numa primeira fase, foram realizadas análises descritivas sobre cada item do instrumento e sobre as dimensões de análise das necessidades dos professores (no âmbito da promoção do desenvolvimento profissional e organizacional, liderança pedagógica e organização do trabalho); num segundo momento, procedeu-se a análises inferenciais em função de características dos sujeitos do estudo, consideradas como variáveis passíveis de explicar alguma variabilidade nas respostas. Os resultados permitem inferir uma valorização da observação e da reflexão entre pares enquanto estratégias de desenvolvimento profissional e organizacional, sendo bastante evidente a necessidade de apoio para cumprirem os desígnios do exercício da vertente de liderança pedagógica da sua ação enquanto diretores, necessidades declaradas de forma mais vigorosa quanto maior é o nível de qualificação académica.

Palavras-chave: Diretores escolares; Liderança pedagógica; Desenvolvimento profissional; Professores principiantes

\section{N T R O D U Ç Ã O}

A investigação sobre desenvolvimento profissional dos professores, os seus ciclos de vida e, mais especificamente, sobre o período inicial das suas vidas profissionais tem sido objeto de uma ampla análise e reflexão. Com efeito, o início do exercício profissional docente vem sendo apontado, na literatura, como um período crítico, sendo recorrentemente experienciado pelo professor principiante como sendo um choque com a realidade (Veenman, 1984).

Em diversos estudos, a fase inicial de imersão 
no contexto profissional surge como uma etapa de adaptação onde sentimentos de entusiasmo e de descoberta coexistem, decorrentes da confrontação com a complexidade do exercício profissional e com a multiplicidade de tarefas e solicitações a que os professores são sujeitos. Não será, pois, surpreendente que as preocupações, nesta fase inicial, sejam prioritariamente direcionadas para a adaptação e sobrevivência e para a integração no grupo profissional (Grimsath, Nordvik, \& Bergsvik, 2008).

Ao que mencionámos, acresce um outro aspeto peculiar na entrada na profissão docente: a atribuição de tarefas e responsabilidades iguais às de um professor experiente. De facto, contrariamente ao que sucede noutras profissões, onde a entrada se dá com a assunção de tarefas que, de forma progressiva, vão sendo mais exigentes e decisivas, "o que acontece sob o olhar dos pares mais experientes, a quem cabe a responsabilidade da sua socialização e indução profissionais (Huberman, 1992), a entrada no ensino é, pelo contrário, repentina e abrupta" (Flores, 1999, p. 172).

Em Portugal, desde o final dos anos 1980, a Lei de Bases do Sistema Educativo (LBSE, Lei $\mathrm{n}^{\circ}$ 46/86) reconhece o direito dos professores à formação permanente, tema retomado no atual Estatuto da Carreira Docente (Portugal, 2013a). De acordo com uma meta-análise sobre a formação inicial de professores, produzida em Portugal, entre 1990 e 2000, a formação permanente deverá preconizar a "criação de um ano de indução profissional dos recémdiplomados para a docência" (Estrela, Esteves, \& Rodrigues, 2002, p. 47).

Mais recentemente, será o Conselho Nacional de Educação (Portugal, 2013b) a reconhecer a importância de a formação contínua se estender "a todos os que, tendo completado a sua formação inicial em cursos destinados ao ensino, aguardam colocação como educadores ou professores, ou têm vínculos laborais precários com o sistema educativo" (p. 68). Estas advertências estão em linha com os estudos e as agendas internacionais, patentes, por exemplo, em relatórios da rede Eurydice (2002) ou em documentos da Comissão Europeia (CE), onde se defende que "seria de perspetivar um sistema global e sem descontinuidades que integrasse a formação inicial, a indução e o aperfeiçoamento profissional contínuo ao longo da carreira, incluindo oportunidades de aprendizagens formais e não formais, informais e não formais" (CE, 2007, p. 13).

No entanto, não obstante as intervenções de atores, situados em diferentes escalas, nacionais e transnacionais, e apesar dos contributos da investigação e do aparente consenso político em torno da necessidade de repensar os processos de indução dos professores na profissão (Kessels, 2010), constata-se que as práticas de suporte a essa fase se mantiveram inalteradas em Portugal (Flores \& Ferreira, 2009). De facto, subsiste o hiato entre o discurso normativo e a sua efetivação, uma vez que a obrigatoriedade de implicar os professores em processos de formação contínua se restringe aos que estão integrados na carreira, através do seu envolvimento num período probatório ${ }^{1}$. Podemos, assim, afirmar que esta efetivação afeta exclusivamente professores que entram na carreira, o que, na larga maioria das vezes, acontece após alguns anos de atividade profissional.

\footnotetext{
1 O período probatório está previsto no artigo 30. ${ }^{\circ}$ do Estatuto da Carreira dos Educadores de Infância e dos Professores dos Ensinos Básicos e Secundário (ECD) aprovado pelo Decreto-Lei n. ${ }^{\circ}$ 139-A/90, de 20 de abril. Nos últimos anos vigoraram disposições que permitiram a dispensa de realização do período probatório, designadamente o Despacho n. ${ }^{\circ}$ 9488/2015, de 20 de agosto, que estabeleceu as condições e procedimentos relativos ao período probatório dos docentes que ingressaram no procedimento concursal anual externo.
} 
Embora os modelos teóricos tendam a perspetivar trajetórias mais ou menos sobrepostas entre os ciclos de vida profissional e os da carreira (Huberman, 1992; Leithwood, 1990; Sikes, 1992), no contexto português encontramos uma particularidade: o afastamento entre ambos, decorrente de um acesso à carreira cada vez mais tardio, muitas vezes vários anos após o início da atividade docente $^{2}$.

Em Portugal, o acompanhamento dos professores principiantes parece estar marcado pela falta de apoio e orientação (Serrazina \& Oliveira, 2002), refém, por um lado, da vontade e interesse de cada professor e, por outro, das dinâmicas que as escolas possam gerar. É nesta última possibilidade que o papel do diretor escolar ganha protagonismo, entendendo-se como decisivo o seu envolvimento na criação e disponibilização de sistemas de acolhimento e apoio aos professores, nos seus anos iniciais de experiência profissional.

Com base nesta premissa, apresentamos um recorte de um estudo quantitativo desenvolvido no âmbito do projeto europeu The Outstanding New Teacher Programme $(\mathrm{ONTP})^{3}$. A questão de pesquisa foi a seguinte: Que necessidades são autoatribuídas pelos diretores de agrupamentos de escolas (AE) portugueses para fazer face ao processo de acolhimento e acompanhamento dos professores, nos primeiros anos de atividade profissional?

O artigo está estruturado, primeiramente, em torno da literatura sobre indução e desenvolvimento profissional, cultura de escola, liderança pedagógica e organização do trabalho. Segue-se a descrição do estudo e dos procedimentos metodológicos, a apresentação dos resultados e, por fim, a análise e discussão dos mesmos, e respetivas conclusões.

\section{INDUÇÃO E DESENVOLVIMENTO PROFISSIONAL}

Sendo reconhecido como uma fase de desenvolvimento profissional (DP), o período de indução é um momento peculiar do percurso profissional dos professores, posterior à formação inicial, e de duração variável. Perspetivado como um processo de socialização, que ocorre no contexto da escola, este deverá possibilitar a assimilação da cultura da organização, bem como a (re)configuração do perfil de desempenho exigível.

É no quadro deste processo de socialização e de ampliação dos saberes profissionais, decorrente da imersão do professor no contexto profissional da escola, que emerge com particular relevância o conceito de DP. Com uma pluralidade de aceções e de variantes semânticas (Brodeur, Deaudelin, \& Bru, 2005; Uwamariya \& Mukamurera, 2005), o DP é entendido ora restringindo-se à formação e capacitação, ora alargando-se a perspetivas mais ecológicas, enquanto processo contínuo de reconfigurações que vão ocorrendo no saber, no agir e no ser do professor, ao longo de toda a trajetória profissional e em interação com o meio (Almeida, 2014). Será nesta última que nos situamos, rejeitando uma perspetiva de DP circunscrita à noção de formação

2 A maioria dos professores em período probatório em 2009/2010 não estava no início da sua atividade; $65 \%$ destes docentes exerciam atividade no sistema por períodos de seis a quinze anos, sem que antes lhes tivesse sido oferecido qualquer apoio ou supervisão como professores iniciantes, ou lhes tivesse sido exigida, ao longo dos seus anos de desempenho, que chegavam a ser, em alguns casos (5\%), superiores a 15, qualquer prestação de prova avaliativa do seu desempenho (Roldão, Reis, \& Costa, 2012).

${ }^{3}$ Um Projeto KA2 Erasmus+: 2014-1-BE02-KA201-000474: http://www.ontp.org/ 
permanente, formação contínua, formação em serviço, reciclagem ou capacitação (Bolam \& McMahon, 2004; Brodeur et al., 2005; Day, 2001; Estrela \& Estrela, 2006; Marcelo, 2009).

Apesar dos usos diferenciados dos conceitos de indução e DP, e da existência de modelos diversos relativos ao ciclo de vida profissional, persiste algum consenso em torno da ideia de que as primeiras experiências profissionais dos professores se revestem de enorme importância para a sua retenção na profissão e para a socialização profissional, bem como no domínio da aprendizagem e DP. Se atentarmos, por exemplo, à socialização profissional, mesmo reconhecendo que tal processo se inicia, ainda que de forma embrionária, na formação inicial (Dubar, 1995; Marcelo, 1991; Zeichner \& Gore, 1990), é nos primeiros anos após a profissionalização que o DP se intensifica, sendo um processo extremamente exigente, que incide na (re)construção da identidade profissional do professor (Carrolo, 1997; Kelchtermans, 2009; Kessels, 2010; Marcelo, 1991, 2009) e na construção de um quadro de referências orientador da ação (Feiman-Nemser, 2008; Marcelo, 1991; Oliveira-Formosinho, 2000; Zeichner \& Gore, 1990).

Reveste-se de enorme relevância para o propósito do nosso trabalho o consenso em torno da ideia de que a intensidade com que é vivida cada etapa na trajetória profissional, bem como o tempo de permanência em cada uma delas, depende de um conjunto de fatores intrínsecos e extrínsecos, que podem acelerar ou inibir os processos de DP (Fessler, 1995; Gonçalves, 1992, 2009; Huberman, 1992; Leithwood, 1990; Sikes, 1992).

O nosso entendimento do processo de DP do professor é socioconstrutivista, de construção pessoal, pela leitura e integração que cada indivíduo faz dos acontecimentos (Hadar \& Brody, 2010), e potenciado pela interação com os outros, o que abre espaço a inúmeras possibilidades de construção de diferentes "professional ways-of-being" (Dall'Alba \& Sandberg, 2006, p. 401). Mais ainda, acolhe a hipótese de se atingirem elevados níveis de proficiência profissional nos primeiros anos de atividade, conjetura já visível nos modelos de Kats (Zabalza, 2007) e de Steffy, Wolf, Pash, e Enz (2000), que defendem que os professores podem atingir níveis de excelência nos primeiros cinco anos de carreira. E assim se dissocia o processo de DP da mera acumulação de anos de experiência per se e se acalenta a ideia de que processos de indução de qualidade poderão antecipar todo o processo de crescimento profissional nas suas diversas dimensões, do ser, do saber e do agir.

\section{CULTURA DE ESCOLA, LIDERANÇA PEDAGÓGICA E ORGANIZAÇÃO DO T R A B A L H O}

Os processos de socialização profissional dos professores principiantes são influenciados por fatores de índole pessoal e de ordem contextual (Cohen-Scali, 2003; Flores, 2004; Harland \& Staniforth, 2006; Marcelo, 1991). Segundo os trabalhos de Zeichner e Gore (1990), o processo de socialização permite ao professor contactar com as crenças, valores e atitudes partilhados pela comunidade profissional em que se insere. A socialização ocorre de três formas distintas: a primeira, funcionalista, em que se pressupõe uma acomodação à cultura pré-existente, garantindo-se a manutenção da cultura vigente; a segunda, interacionista, em que se admite a reciprocidade de influências entre os novos professores e as organizações, o que nos remete para alguma flexibilidade individual $\mathrm{e}$ organizacional consentânea com a possibilidade de despontarem diferentes matizes de atuação dos professores; a terceira, crítica, que resulta de um questionamento da cultura pré-existente, podendo redundar em processos de rutura ou de renovação organizacional. 
Perfilhando o pensamento de Marcelo (2009), consideramos que o DP "está diretamente relacionado com os processos de reforma da escola, na medida em que este é entendido como um processo que tende a reconstruir a cultura escolar e no qual se implicam os professores enquanto profissionais" (p. 11). Assim sendo, pressupõe-se um processo de DP iniciado ou acelerado pela imersão num contexto organizacional, com o qual o professor interatua e que, embora vivido na primeira pessoa, é fortemente marcado pelos contextos organizacionais, sociais e histórico-políticos em que o professor se movimenta (Day, 2001; Huberman, 1992). Este influxo é, porém, bidirecional, no sentido em que o DP é tributário do modo como os seus atores contribuem para a aprendizagem e transformação institucional (Cruz, 2006; Day, 2001; Fullan, 1990; Hargreaves, 1998; Marcelo, 2009; Morais \& Medeiros, 2007; Villegas-Reimers, 2003; Zabalza, 2007).

Como vimos, em Portugal, o apoio aos novos professores não se encontra estruturado, subsistindo um vazio entre a formação inicial e a formação contínua, esta última direcionada aos professores de carreira, o que resulta em duas opções: 1) cada professor principiante sobrevive por si só, dependendo da capacidade e iniciativa pessoal, ou 2) as escolas e seus diretores chamam a si esta missão, numa lógica de conjugação de esforços individuais e coletivos. A última opção afigura-se vantajosa para ambos, professores e escola, uma vez que uma "escola eficiente tem que ser educativa para os seus professores" (Shulman, 2004, p. 505) e uma "organização que aprende" é uma organização que desenvolve processos de reflexão, que organiza, avalia e reflete sobre a sua ação como uma oportunidade para aprender (Argyris \& Schön, 1996).

Então, "se se quer facilitar o DP temos que perceber o processo mediante o qual os professores crescem profissionalmente, bem como as condições que ajudam e promovem esse crescimento" (Marcelo, 2009, p. 16), para que este possa ocorrer o mais cedo possível. Ancorados na premissa da complementaridade entre a dimensão individual e a dimensão organizacional e na ideia de que é possível promover o DP dos docentes, intervindo precocemente no seu exercício profissional, aduzimos à importância que os processos de indução devem ter na cultura organizacional e nas preocupações de quem tem a seu cargo a liderança pedagógica da escola: o diretor escolar. Efetivamente, num contexto de inexistência de sistemas formais de indução de professores, o diretor escolar vê-se na contingência de o assumir ou então deixar que as leis de seleção natural atuem, metaforizando os princípios das leis darwinistas.

Dirigir uma escola é um empreendimento exigente. No contexto português, consubstanciase no singular, na figura do diretor (Portugal, 2008), o que constitui um veículo de expressão de uma determinada cultura escolar (Torres, 2013), unipessoal, "entendida como a expressão de um líder individual, de um 'rosto' ou "executivo eficaz" (Lima, 2014, p. 1076), o qual atua num contexto ambíguo, porque marcado por modos de regulação da educação híbridos, numa tensão entre "o modelo de um governo burocrático, controlador e regulamentador" e "o modelo da governança que faz apelo à negociação e à participação dos diferentes atores" (Barroso, 2011, p. 14).

A literatura sobre o diretor escolar tem focado a complexidade do cargo e as inúmeras e intrincadas transformações a que vem estando sujeito (Cattonar, 2006; Earley \& Bubb, 2013; Horng, Klasik, \& Loeb, 2009; O’Malley, Long, \& King, 2015), intervindo na dimensão formal da escola, através da coordenação de estruturas e processos, de modo a alcançar as metas do seu Projeto Educativo, mas igualmente intervindo ao nível relacional, atentando às necessidades sentidas pelos professores na escola, o que depende da criação de culturas favoráveis a processos sustentadores de DP. O comprometimento, a visão estratégica e a capacidade de liderança do diretor escolar são facilitadores da promoção de processos de indução e de DP, os quais obedecem a variáveis pessoais e profissionais dos professores e a variáveis contextuais do ambiente político-social em que atuam. 
Não obstante a literatura revelar que os diretores escolares não centram a sua ação na liderança pedagógica (Flores, Mendes, \& Velosas, 2013), as dimensões da organização do trabalho e do exercício da liderança pedagógica têm vindo a ser apontadas como vitais para a melhoria da qualidade das práticas e dos resultados dos alunos (Horng \& Loeb, 2010). A redefinição do papel do diretor na condução do projeto educativo e o envolvimento da escola em seu redor (Cattonar, 2006) ganha especial relevo, destacando-se o potencial congregador do diretor, que advém da natureza relacional, comunicacional, horizontal, e não explícita, da ativação de processos de liderança, e que se traduz em modos mais subtis de coordenar os atores escolares. A liderança, assim compreendida, como "processo pelo qual um indivíduo influencia um grupo de indivíduos para alcançar um objetivo comum" (Northouse, 2010, p. 3), é construída em (cada) contexto, no âmbito de cada comunidade, e opera pela persuasão, pela motivação e o convencimento, de modo a que se alcancem as metas por todos compartilhadas (Yukl, 2002).

Reconhece-se hoje que o sucesso dos líderes escolares depende de conseguir melhorar a qualidade das práticas institucionais (Elmore, 2006) e da sua capacidade organizativa, ao estimular os atores escolares para a consecução dos objetivos organizacionais (Whithaker, 2000). A literatura aponta como núcleo duro de práticas de liderança nas escolas: fixar 'direções' para o trabalho escolar; desenvolver as pessoas; desenvolver a organização e responder aos desafios e oportunidades do ambiente organizacional (Leithwood \& Riehl, 2003). Sendo assim, torna-se evidente a responsabilidade do diretor em promover dispositivos e culturas de apoio aos professores durante o seu processo de indução (Stansbury, 2001; Sunde \& Ulvik, 2014). Vários autores consideram inclusivamente tratar-se de uma obrigação moral dos mesmos providenciar sistemas de apoio de elevada qualidade (Greenfield, 1995; Wischkaemper, 2005). Estes sistemas de apoio poderão contribuir também para minorar o mal-estar docente característico da fase inicial da vida profissional
(Chubbuck, Clift, Allard, \& Quinlan, 2001; DeWert, Babinski, \& Jones, 2003; Kessels, 2010; Molner Kelley, 2004) e para a retenção na carreira (Blaya \& Baudrit, 2005; Marable \& Raimondi, 2007), contrariando a tendência crescente de abandono precoce da profissão que se verifica em diversos países da Europa (CE, 2010; OECD, 2005).

Deste modo, tornando-se evidente a importância do diretor escolar na organização do trabalho docente e na mobilização dos professores (recém-chegados ou mais experientes), cumprenos indagar sobre as necessidades sentidas pelos diretores escolares portugueses relativamente ao apoio à fase da indução.

\section{Objetivos do ESTUdo E Eixos de} ANÁLISE

Partindo do entendimento de que necessidades "são sempre relativas aos indivíduos e aos contextos e decorrem de valores, pressupostos e crenças" (Esteves \& Rodrigues, 1993, p. 12), o estudo teve como objetivo perceber aquelas que são autoatribuídas pelos diretores de Agrupamentos de Escola/Escolas portugueses/ as para fazer face ao processo de indução de professores, nos cinco primeiros anos de atividade profissional, estejam ou não integrados na carreira. Assim, foram definidos três eixos de análise que, a partir do quadro teórico discutido, se consideraram dimensões da intervenção do diretor e que se vieram a constituir como dimensões concetuais do instrumento de recolha de dados, a saber:

a. o desenvolvimento profissional e organizacional (integrando-se aqui o papel que o diretor e a escola têm como promotores do DP dos professores, bem como a escola enquanto objeto em transformação por quem aí desenvolve a sua atividade profissional);

b. a organização do trabalho (reconhecendose aqui que o modo como o diretor organiza o trabalho na e da escola para responder às necessidades individuais e coletivas pode inibir ou potenciar o crescimento e a 
aprendizagem dos professores); e

c. a liderança pedagógica (considerando-se que esta dimensão do trabalho do diretor pode estimular ou inibir um trabalho de cariz mais colaborativo entre os pares, bem como influir nas práticas de ensinoaprendizagem desde os anos iniciais da atividade profissional dos professores).

\section{Método}

\subsection{INSTRUMENTO}

$\mathrm{O}$ artigo resulta de um estudo de natureza quantitativa, centrado na aplicação de um questionário eletrónico aos diretores escolares do território nacional continental português. O instrumento aplicado foi um questionário de opinião (Ghiglione \& Matalon, 1997), construído para o estudo, com base na revisão de literatura, e recorrendo-se à consulta de especialistas para a sua validação. Com o intuito de perceber que necessidades são apontadas pelos diretores escolares para promover e encorajar práticas de integração e apoio aos professores principiantes, definiram-se as seguintes dimensões: desenvolvimento profissional e organizacional (19 itens); organização do trabalho (9 itens) e liderança pedagógica (14 itens). Selecionou-se, ainda, um conjunto de variáveis independentes (género, idade, qualificações académicas e profissionais, formação específica em administração e gestão escolar e anos de experiência como diretor/na direção), cuja interferência nas respostas se procurou estudar.

Foram dadas as seguintes instruções de preenchimento: "No exercício da sua função, o diretor assume diferentes papéis. Alguns desses papéis prendem-se com o acolhimento e apoio aos professores recém-formados/principiantes (até cinco anos de serviço). Pedimos-lhe que, utilizando a escala de 1 a 5 , em que 1 corresponde a nada e 5 a muitíssimo, nos dê a sua opinião sobre as áreas em que gostaria de ter apoio (aconselhamento, formação, etc.)”.

A análise à consistência interna do instrumento, através do cálculo do alfa de Cronbach, revelou índices de confiabilidade bastante elevados (ver tabela 3): .94 para a dimensão de liderança pedagógica, .94 para a dimensão da organização do trabalho e .96 para a dimensão de desenvolvimento profissional e organizacional.

\subsection{PROCEDIMENTOS DE RECOLHA DE DADOS E PARTICIPANTES}

Os questionários foram enviados, por via eletrónica, para o total de 713 AE de Portugal continental $^{4}$. Foram devolvidos, devidamente preenchidos, 125 questionários, o que perfaz uma taxa de resposta de $17,5 \%$. Dado não conhecermos todas as características da população estudada, não podemos considerar que se trata de uma amostra representativa.

Em função das variáveis selecionadas para o estudo, a amostra apresenta as seguintes características: a larga maioria é do género feminino (79\%) e tem maioritariamente mais de 50 anos $(65,6 \%)$, seguindo-se um segundo grupo, entre os 41 e os 50 anos (32,8\%); apenas $1,6 \%$ se situa entre os 31 e os 40 anos. Para efeitos de análise de dados e devido à baixa representação do grupo de participantes com idades compreendidas entre os 31 e 40 anos, agrupámos os sujeitos em dois grupos: mais de 50 anos e menos de 50 anos. É de salientar que a maioria dos respondentes detém o grau de licenciado (60,8\%), 38,4\% são mestres e apenas $0,8 \%$ tem doutoramento. Para efeitos de testagem das diferenças entre respondentes, agrupámos os sujeitos em dois grupos: um grupo incluindo os licenciados e outro integrando os mestres e doutores. A larga maioria dos diretores que responderam ao questionário tem formação específica na área da administração escolar $(86,4 \%)$.

Em relação à experiência docente, a grande maioria acumula uma experiência superior a 20

4 Em Portugal existem 811 unidades orgânicas, das quais 713 são agrupamentos de escolas e 98 escolas não agrupadas (Ministério da Educação e Ciência, 2017). 
anos $(82,4 \%)$, sendo que apenas $14,4 \%$ têm entre 16 e 20 anos de experiência e somente 3,2\% têm menos de 16 anos de experiência. Relativamente à experiência como diretor/na direção, encontramos alguma dispersão: $44 \%$ dos sujeitos têm mais de dez anos de experiência, mas 29,6\% têm apenas entre um e cinco anos de experiência e $26,4 \%$ têm entre seis e dez anos.

\subsection{Procedimentos De ANÁlise DE DA D O S}

Os dados foram analisados através de uma abordagem quantitativa e com recurso ao software estatístico IBM SPSS versão 22. Foram conduzidos dois procedimentos de análise de dados. Primeiramente, procedemos a análises descritivas sobre cada item do instrumento e sobre as dimensões de análise das necessidades dos diretores (através de medidas de tendência central e dispersão). Num segundo momento, procedemos à análise inferencial considerando algumas características dos diretores (variáveis independentes). Utilizámos testes paramétricos t-Student e análise de variância (ANOVA), pois embora as variáveis não seguissem distribuição normal em todos os casos, os valores de assimetria e curtose (ver tabela 3) apresentam-se

TA B ELA 1

Itens do Questionário com Maior Pontuação Média como bastante satisfatórios para a utilização de metodologias de análise mais robustas (Marôco, 2018).

\section{Apresentação dos resultados}

Na tabela 1 são apresentados os dez itens que correspondem às principais necessidades que os diretores se autoatribuem para fazer face ao processo de acolhimento e acompanhamento dos professores. Por contraste, a tabela 2 reúne os dez itens que correspondem às questões percebidas pelos diretores como aspetos em que sentem menos necessidade de apoio.

Como se pode observar na tabela 1,0 item que mais se destaca refere-se à necessidade de apoio para implementar dispositivos de observação/ supervisão da prática letiva $(M=3.97)$, integrando a dimensão desenvolvimento profissional e organizacional (DPO). Os itens seguintes inserem-se na dimensão liderança pedagógica, estando relacionados com a promoção de práticas de diferenciação pedagógica, coordenação e gestão do currículo, a promoção da preparação dos alunos para a sociedade futura, a análise e reconfiguração dos ambientes de aprendizagem dos alunos e a promoção do conhecimento e reflexão sobre gestão da interação em sala de aula.

\begin{tabular}{|l|l|l|}
\hline Item & $M$ & $D P$ \\
\hline 15. Implementar um dispositivo de supervisão/observação da prática letiva & 3.97 & 1.02 \\
\hline 5. Promover práticas de diferenciação pedagógica & 3.82 & 1.11 \\
\hline 4. Coordenar a gestão vertical e horizontal do currículo escolar & 3.71 & 1.16 \\
\hline 9. Promover a preparação dos alunos para a sociedade futura & 3.67 & 1.01 \\
\hline 12. Estimular a análise e reconfiguração dos ambientes de aprendizagem dos alunos & 3.66 & 0.96 \\
\hline 2. Promover o conhecimento e reflexão sobre gestão da interação em sala de aula & 3.58 & 1.05 \\
\hline 42. Criar espaços de reflexão crítica do professor sobre o seu próprio trabalho & 3.57 & 1.15 \\
\hline 30. Promover o comprometimento com a missão e objetivos da escola & 3.54 & 1.07 \\
\hline 3. Promover a definição/reflexão sobre o sistema de avaliação e classificação dos alunos & 3.53 & 1.13 \\
\hline 7. Promover práticas de apoio ao crescimento individual do aluno & 3.46 & 1.07 \\
\hline
\end{tabular}


TA B ELA 2

Itens do Questionário com Menor Pontuação Média

\begin{tabular}{|l|l|l|}
\hline Item & $M$ & $D P$ \\
\hline $\begin{array}{l}\text { 19. Promover a participação dos novos professores em tarefas fora da sala de aula (tomar } \\
\text { conta dos alunos durante o intervalo, festas da escola, viagens, reuniões, etc.) }\end{array}$ & 2.99 & 1.16 \\
\hline 37. Planear atividades que favoreçam a integração dos novos professores na cultura da escola & 2.96 & 1.17 \\
\hline $\begin{array}{l}\text { 24. Garantir de um sistema de apoio aos novos professores no trabalho a desenvolver com } \\
\text { representantes do mundo laboral }\end{array}$ & 2.91 & 1.08 \\
\hline $\begin{array}{l}\text { 27. Garantir sistema de apoio aos novos professores no trabalho a desenvolver em } \\
\text { articulação com técnicos educativos (psicólogos, técnicos de serviço social, animadores...) }\end{array}$ & 2.91 & 1.02 \\
\hline $\begin{array}{l}\text { 20. Explicitar procedimentos relativos a tarefas administrativas (cartas informativas, } \\
\text { relatórios, transferências de alunos para outros grupos ou escolas, agendas de trabalho) }\end{array}$ & 2.9 & 1.04 \\
\hline $\begin{array}{l}\text { 22. Garantir a existência de canais de comunicação entre os professores recém-formados e a } \\
\text { direção }\end{array}$ & 2.87 & 1.13 \\
\hline $\begin{array}{l}\text { 25. Garantir sistema de apoio aos novos professores no trabalho a desenvolver com } \\
\text { representantes da vida cultural }\end{array}$ & 2.87 & 1.05 \\
\hline 23. Garantir a existência de um interlocutor da escola com os professores recém-formados & 2.81 & 1.16 \\
\hline 11. Promover a equidade entre sexos & 2.80 & 1.16 \\
\hline $\begin{array}{l}\text { 26. Garantir sistema de apoio aos novos professores no trabalho a desenvolver com o corpo } \\
\text { docente e outros funcionários da escola }\end{array}$ & 2.74 & 1.09 \\
\hline
\end{tabular}

Surgem, em seguida, dois itens da dimensão DPO, nomeadamente a necessidade de apoio sentida para criar espaços de reflexão crítica do professor sobre o seu próprio trabalho e para promover o comprometimento com a missão e objetivos da escola. Seguem-se, novamente, necessidades associadas às questões da liderança pedagógica, que se prendem com a definição/reflexão sobre o sistema de avaliação e classificação dos alunos e de promoção de práticas de apoio ao crescimento individual do aluno.

A dimensão liderança pedagógica assume, então, um lugar destacado nas necessidades declaradas pelos diretores, pois das dez necessidades prioritárias, sete pertencem a esta dimensão. As restantes três situam-se no âmbito da promoção do DPO, com especial destaque para a necessidade de apoio para a implementação de dispositivos de observação/ supervisão da prática letiva. É de assinalar que, nesta primeira ordenação, nenhuma das necessidades declaradas surge relacionada com a dimensão de organização do trabalho.

Observando a tabela 2, verificamos que os diretores declaram menos necessidade de auxílio para garantir sistemas de apoio aos novos professores no trabalho a desenvolver com o corpo docente e outros funcionários da escola, na promoção da equidade entre géneros e na articulação do trabalho com outros atores educativos. Os restantes itens menos expressivos estão relacionados com a dimensão da organização do trabalho.

Em síntese, e considerando as três dimensões do estudo, verifica-se que os diretores assinalam sobretudo necessidades na dimensão da liderança pedagógica $(M=3.41)$, seguindo-se a dimensão do DPO $(M=3.19)$ e, por último, a referente à organização do trabalho $(M=3.07)$ (ver tabela 3). Também de assinalar que é nesta última que verificamos uma maior variação de respostas.

A análise em torno da possível interferência de algumas características individuais no modo como os diretores se manifestam face às necessidades de apoio (ver tabela 4) revelou, desde logo, não haver diferenças estatisticamente significativas decorrentes do género: $t(123)=0.55$, $p=.583$ para a dimensão liderança pedagógica, 
TA B EL A 3

Análise Descritiva e Consistência Interna para as Três Dimensões

\begin{tabular}{|c|c|c|c|c|c|c|}
\hline & $M$ & $D P$ & Amp. & Assimetria & Curtose & $\begin{array}{c}\text { Alpha de } \\
\text { Cronbach }\end{array}$ \\
\hline Liderança pedagógica & 3.41 & 0.81 & $3.00-3.96$ & -0.79 & 0.71 & 0.94 \\
\hline $\begin{array}{c}\text { Organização do trabalho } \\
\text { Desenvolvimento profissional e } \\
\text { organizacional }\end{array}$ & 3.07 & 0.97 & $2.33-3.89$ & -0.40 & -0.59 & 0.94 \\
\hline \begin{tabular}{c} 
(a) \\
\hline
\end{tabular} & 0.89 & $2.53-3.89$ & -0.36 & -0.41 & 0.96 \\
\hline
\end{tabular}

TA B E L A 4

Análise Descritiva Considerando as Dimensões e Variáveis Individuais dos Diretores

\begin{tabular}{|c|c|c|c|c|c|c|}
\hline & \multicolumn{2}{|c|}{$\begin{array}{c}\text { Liderança } \\
\text { pedagógica }\end{array}$} & \multicolumn{2}{|c|}{$\begin{array}{c}\text { Organização do } \\
\text { trabalho }\end{array}$} & \multicolumn{2}{|c|}{$\begin{array}{c}\text { Desenvolvimento } \\
\text { profissional e organizacional }\end{array}$} \\
\hline & $M$ & $D P$ & $M$ & $D P$ & $M$ & $D P$ \\
\hline \multicolumn{7}{|l|}{ Idade } \\
\hline Menos de 50 anos & 3.23 & 0.86 & 2.95 & 1.09 & 3.01 & 1.01 \\
\hline Mais de 50 anos & 3.50 & 0.78 & 3.13 & 0.90 & 3.28 & 0.80 \\
\hline \multicolumn{7}{|l|}{ Género } \\
\hline Feminino & 3.44 & 0.76 & 3.18 & 0.97 & 3.26 & 0.88 \\
\hline Masculino & 3.35 & 0.91 & 2.87 & 0.93 & 3.07 & 0.89 \\
\hline \multicolumn{7}{|l|}{ Formação académica } \\
\hline Licenciatura & 3.27 & 0.83 & 2.98 & 0.10 & 3.10 & 0.82 \\
\hline Mestrado ou superior & 3.61 & 0.76 & 3.20 & 0.15 & 3.32 & 0.98 \\
\hline \multicolumn{7}{|c|}{ Formação em administração escolar } \\
\hline Sim & 3.49 & 0.72 & 2.67 & 0.09 & 2.76 & 0.26 \\
\hline Não & 2.87 & 1.14 & 3.13 & 0.28 & 3.26 & 0.08 \\
\hline \multicolumn{7}{|l|}{ Experiência como diretor } \\
\hline 1 - 5 anos de experiência & 3.19 & 1.06 & 2.91 & 1.09 & 2.93 & 1.06 \\
\hline 6-10 anos de experiência & 3.71 & 0.65 & 3.48 & 0.78 & 3.61 & 0.68 \\
\hline $\begin{array}{l}\text { Mais de } 10 \text { anos de } \\
\text { experiência }\end{array}$ & 3.37 & 0.66 & 2.93 & 0.92 & 3.11 & 0.78 \\
\hline
\end{tabular}


$t(123)=1.78, \quad p=.077$ para a dimensão organização do trabalho e $t(123)=1.14, p=.257$ para a dimensão desenvolvimento profissional e organizacional; também não revelou diferenças para a idade dos diretores: $t(123)=-1.75, p=.083$, para a dimensão liderança organizacional, $t(123)=-0.98, \quad p=.331$, para a dimensão organização do trabalho e $t(123)=-1.67, p=.097$, e para a dimensão desenvolvimento profissional e organizacional. No entanto, foram detetadas diferenças decorrentes da formação académica, da obtenção de formação especializada na área da gestão e administração escolar e dos anos de experiência no cargo.

Assim, relativamente à formação académica, observam-se diferenças estatisticamente significativas no posicionamento dos diretores relativamente à dimensão liderança pedagógica - $t(123)=-2.31, p=.023$-, verificando-se que os detentores de graus académicos mais elevados demonstram uma preocupação superior nesta dimensão $(M=3.61)$. Ainda nesta dimensão verificou-se que os diretores com formação em gestão e administração escolar expressam uma maior necessidade de apoio face aos que não a têm: $(M=3.49), \quad t(123)=-2.99$, $p=.003$. Ainda, verificou-se também que estes diretores manifestam uma maior preocupação relativamente à dimensão desenvolvimento profissional e organizacional $(M=3.26)$, quando comparados com diretores sem formação em gestão e administração: $t(123)=-2.16, p=.033$. No que diz respeito à dimensão organização do trabalho, não foram encontradas diferenças estatisticamente significativas, tanto em função da formação em administração escolar $t(123)=-1.76, p=.080$-, bem como em função da formação académica $-t(123)=-1.34, p=.181$. Por último, não se verificaram igualmente diferenças estatisticamente significativas para a formação académica relativamente à dimensão desenvolvimento profissional e organizacional: $t(123)=-1.34, p=.181$.

Já a variável anos de experiência como diretor tem impacto significativo nas três dimensões: $F(2,124)=3.89, p=.023$ na dimensão liderança pedagógica, $F(2,124)=4.34, p=.015$ na dimensão organização do trabalho, $F(2,124)=4.37, p=.003$ na dimensão desenvolvimento profissional e organizacional. Os que revelam maior necessidade na dimensão liderança pedagógica são os que têm entre seis e dez anos de experiência $(M=3.71)$. Relativamente à dimensão organização do trabalho e à dimensão do desenvolvimento profissional e organizacional existem diferenças significativas entre o grupo de diretores com experiência entre os seis e os dez anos e os restantes grupos, sendo que os primeiros expressam maior necessidade nestas dimensões ( $M=3.48$ e $M=3.61$, respetivamente).

\section{ANÁlise e discussão dos RESUltados}

Resultando de um projeto mais vasto, centrado na indução de professores em início de atividade, o presente estudo teve a singularidade de se focar nas necessidades percebidas pelos diretores escolares quanto ao apoio a dar aos professores na fase de indução. Como se viu, em Portugal subsiste um hiato entre a dimensão discursiva e a sua efetivação, a propósito do acolhimento a professores na fase inicial da atividade profissional. A inexistente regulamentação do período de indução (ME, 2007) pelo regulador nacional (Estado) remete unicamente para as escolas e para o diretor a responsabilidade de construir sistemas de acolhimento e de apoio.

Numa primeira análise, e surpreendentemente, percebemos que a grande preocupação declarada pelos diretores diz respeito à efetivação do exercício de uma liderança pedagógica, o que vem sendo referenciado, na literatura, como uma área de atuação onde tradicionalmente os diretores se envolvem pouco, pois é delegada essencialmente no corpo docente. Barroso e Carvalho (2009) chamam a atenção para o facto de a chamada 'crise da escola' ser acima de tudo pedagógica e organizacional e influenciada pela interação do ambiente educacional/social e pelas opções políticas que regulam. A Organização para a Cooperação e Desenvolvimento Económico (OCDE, 2005) preconiza a importância de acolher e apoiar os professores principiantes, estabelecendo condições favoráveis para o 
seu DP, na convicção de que é possível atingir níveis de proficiência profissional elevados nos primeiros anos de atividade (Steffy et al., 2000; Zabalza, 2007).

Em Portugal, se tivermos presentes estudos anteriores, que identificavam que "em média, os líderes das escolas portuguesas assumem menos responsabilidades de liderança do que os seus pares" (Flores et al., 2013, p. 388), estes resultados são inesperados e porventura evidenciarão uma orientação para a mudança e uma vontade de implicação em processos de adaptação e inovação na organização escolar (Murillo, 2003). Também se percebe, pelas necessidades declaradas, que se acredita que tal investimento terá reflexos positivos nos processos de ensino-aprendizagem (Cochran-Smith, 2006; Darling-Hammond, 2006; Rivkin, Hanushek, \& Kain, 2005). Como se viu, a criação de processos e de espaços de reflexão sobre as práticas (Cherian \& Daniel, 2008; Leithwood, Day, Sammons, Harris, \& Hopkins, 2006) e o reforço do comprometimento dos professores com a missão e os objetivos da escola preocupam, igualmente, os diretores, o que está alinhado com uma elevada preocupação com os processos de socialização profissional dos professores principiantes presente em diversos estudos (Cohen-Scali, 2003; Flores, 2004; Harland \& Staniforth, 2006; Marcelo, 1991).

Outra área em que os diretores dizem sentir maior carência reporta-se à implementação de dispositivos de observação/supervisão da prática letiva, o que pode refletir per se uma preocupação ou uma valorização destas práticas como ferramentas privilegiadas de DP, indo ao encontro do reconhecimento, recorrentemente exortado na literatura, das potencialidades da observação entre pares (Alarcão, 1996; Alarcão \& Roldão, 2008; Butler, 2005; Gonçalves, 2009). Porém, acreditamos que esta valoração da observação de aulas não é imune à relevância que lhe é atribuída por organizações internacionais como a OCDE, que descreve esta questão, no contexto português, da seguinte forma: "Within schools, there is no culture of classroom observation and little tradition of peer evaluation, feedback and the sharing of good practice"
(Santiago, Roseveare, Van Amelsvoort, Manzi, \& Matthews, 2009, p. 3), concluindo: "Therefore, we concur with the Portuguese system in underlining the role of classroom observation" (Santiago et al., 2009, p. 7). A inexistência de uma cultura de observação de aulas é, também, assinalada em estudos nacionais, por exemplo, a propósito do Programa de Avaliação Externa das Escolas, desenvolvido pela Inspeção da Educação, que a tem evidenciado como problema (Conselho Nacional de Educação, 2015).

Inscrita no pensamento dos diretores, esta necessidade será porventura, ainda, resultado da instauração (e intensificação) de um ambiente institucional fomentador de práticas de observação de aulas (Fialho, Saragoça, Silvestre, Correia, \& Gomes, 2014), a que a administração educativa parece ter aderido, e que decorrerão, em última análise, de processos mais amplos e dos efeitos que advêm das dinâmicas de regulação transnacional (Carvalho, 2012) em que a OCDE intervém como criadora e legitimadora de ideias (Carvalho, 2012; Mahon \& MacBride, 2008), concorrendo para a construção de narrativas educacionais sobre o governo da educação (Carvalho, Costa, \& Gonçalves, 2017).

Os resultados do estudo permitem, além disso, evidenciar que o posicionamento dos diretores depende, de alguma forma, de características inerentes à sua formação e experiência. É de destacar a importância da variável 'formação' (seja através da obtenção de graus superiores, seja por via da formação especializada na área da administração $e$ gestão escolar), uma vez que a sinalização de necessidades no âmbito da dimensão liderança pedagógica é significativamente superior nos grupos com formação académica mais elevada ou especializada. Este dado permite aventar a hipótese de que a formação proporciona aos diretores um olhar mais amplo e crítico relativo aos contextos e à sua própria ação, através de novos quadros de referência, o que leva a uma preocupação que vai para além das tarefas técnicas de administração e gestão, "cada vez mais complexas e em maior número" (Bolívar, 2006, p. 91), alargando-se à dimensão pedagógica da sua 
ação, tradicionalmente menos considerada pelos diretores (Bolívar \& Moreno, 2006; Elmore, 2000; Waters \& Grubb, 2004).

Quanto aos anos de experiência no cargo, são os diretores com seis a dez anos de experiência os que manifestam maior necessidade de apoio, quando comparados com os restantes grupos. Será interessante, em estudos posteriores, aprofundar a relação entre a experiência no cargo e as necessidades manifestadas, uma vez que, contrariamente ao que seria expectável, não é o grupo mais inexperiente a destacar-se face aos outros.

Uma das limitações do estudo decorreu da falta de acesso a uma caracterização dos diretores (idade, género, etc.).

\section{Conclus Ão}

As necessidades declaradas pelos diretores traduzem uma preocupação, não tanto com a dimensão gestionária do cargo que ocupam (técnicas, funções e tarefas), mas com processos de liderança, no sentido da influência social, de motivação e envolvimento, na estruturação das atividades e relações num grupo e na organização (Yukl, 2002). Os diretores parecem estar concentrados em desenvolver processos de liderança situada, que obriguem a um conhecimento do projeto de escola, comprometendo-se com a identidade (única) da escola que dirigem (bem visível na questão da missão e dos objetivos da escola).

Deforma mais ou menosinduzida por processos regulatórios transnacionais (Organização para a Cooperação e Desenvolvimento Económico, União Europeia) e nacionais (Conselho Nacional de Educação, Ministério da Educação), as necessidades autoproclamadas são reveladoras de um entendimento do cargo de diretor consonante com "uma liderança pedagógica estratégica, proativa, efetivamente centrada nas aprendizagens dos alunos, na promoção do desenvolvimento profissional dos professores e numa cultura institucional de reflexão sobre as práticas de ensino" (Costa, 2015, p. 254), na esteira do que Leithwood e Riehl (2003) advogam como base do sucesso dos diretores escolares. Mais ainda, os resultados apurados indiciam uma consciencialização dos diretores relativamente à necessidade de assumir um papel ativo na promoção do DP dos professores principiantes, dimensão até agora pouco visível na literatura.

\section{REFERENCIAS}

Alarcão, I. (Ed.). (1996). Formação reflexiva de professores. Estratégias de supervisão. Porto: Porto Editora.

Alarcão, I., \& Roldão, M. C. (2008). Supervisão. Um contexto de desenvolvimento profissional dos professores. Mangualde: Edições Pedago.

Almeida, M. (2014). Trajetórias no desenvolvimento profissional docente no ensino superior: Fatores condicionantes. Revista Portuguesa de Pedagogia, 48(2), 61-85.

Argyris, C., \& Schön, D. (1996). Organizational learning. Reading, MA: Addison Wesley.

Barroso, J. (2011). Direcção de escolas e regulação das políticas: Em busca do unicórnio. In A. Neto-Mendes, J. A. Costa, \& A. Ventura (Orgs.), A emergência do director da escola: Questões políticas e organizacionais (pp. 1121). Aveiro: Universidade de Aveiro.

Barroso, J., \& Carvalho, L. M. (2009). La gestión de centros de enseñanza obligatoria en Portugal. In J. Gairín (Coord.), La gestión de centros de enseñanza obligatoria en Iberoamérica (pp. 125-137). s/l.: RedAGE.

Blaya, C., \& Baudrit, A. (2005). ¿Entre necesidad y factibilidad? El mentorado de profesores al principio de su carrera para el manejo de situaciones difíciles. Revista Mexicana de Investigación Educativa, 10(26),765-786.

Bolam, R., \& McMahon, A. (2004). Literature, definitions and model: Towards a conceptual map. In C. Day (Ed.), International handbook on the continuing professional development of teachers (pp. 33-64). Berkshire: McGraw-Hill. 
Bolívar, A. (2006). A liderança educacional da direcção escolar em Espanha: Entre a necessidade e a (im)possibilidade. Revista do Fórum Português de Administração Educacional, 6, 76-94.

Bolívar, A., \& Moreno, J. M. (2006). Between transaction and transformation: The role of school principals as education leaders in Spain. Journal of Educational Change, 7(1-2), 19-31.

Brodeur, M., Deaudelin, C., \& Bru, M. (2005). Introduction : Le développement professionnel des enseignants: Apprendre à enseigner pour soutenir l'apprentissage des élèves. Revue des Sciences de l'Éducation, 31(1), 5-14.

Butler, D. (2005). L'autorégulation de l'apprentissage et la collaboration dans le développement professionnel des enseignants. Revue des Sciences de l'Éducation, 31(1), 55-78.

Carrolo, C. (1997). Formação e identidade profissional dos professores. In M. T. Estrela (Ed.), Viver e construir a profissão docente (pp. 21-50). Porto: Porto Editora.

Carvalho, L. M. (2012). The fabrications and travels of a knowledge-policy instrument. European Educational Research Journal, 11(2), 172-188.

Carvalho, L. M., Costa, E., \& Gonçalves, C. (2017). Fifteen years looking at the mirror: On the presence of PISA in education policy processes (Portugal, 2000-2016). European Journal of Education, 00, 1-13. doi:10.1111/ ejed. 12210

Cattonar, B. (2006). Evolução do modo de regulação escolar e reestruturação da função de diretor de escola. Educação em Revista, 44, 185-208.

Cherian, F., \& Daniel, Y. (2008). Principal leadership in new teacher induction: Becoming agents of change. International Journal of Education Policy and Leadership, 3(2), 1-11.

Chubbuck, S. M., Clift, R. T., Allard, J., \& Quinlan, J. (2001). Playing it safe as a novice teacher: Implications for programs for new teachers. Journal of Teacher Education, 52, 365-376. doi:10.1177/0022487101052005003
Cochran-Smith, M. (2006). Policy, practice, and politics in teacher education. Thousand Oaks, CA: Corwin Press.

Cohen-Scali, V. (2003). The influence of family, social, and work socialization on the construction of the professional identity of young adults. Journal of Career Development, 29,237-249. doi:10.1177/089484530302900402

Comissão Europeia. (2010). Developing coherent and system-wide induction programmes for beginning teachers: A handbook for policymakers. European Commission Staff Working Document SEC (2010) 538 final. Directorate-General for Education and Culture. Disponível em http://ec.europa. eu/assets/eac/education/policy/school/doc/ handbook0410 en.pdf

Comissão Europeia. (2007). Melhorar a qualidade da formação académica e profissional dos docentes. Comunicação da Comissão Europeia ao Conselho e ao Parlamento Europeu. Disponível em: http://eur-lex.europa.eu/legalcontent/PT/TXT/?uri=LEGISSUM:c11101

Conselho Nacional de Educação. (2015). Avaliação externa das escolas. Disponível em: http://www.cnedu.pt/content/edicoes/ seminarios e coloquios/LIVROCNE AVALIA \%C $3 \% 87 \% \mathrm{C} 3 \% 830$ EXTERNA DAS ESCOLAS.pdf

Costa, E. (2015). Da liderança pedagógica do diretor escolar aos processos de reconfiguração organizacional da escola. In Conselho Nacional de Educação, Estado da educação 2014 (pp. 254-261). Lisboa: Conselho Nacional de Educação.

Cruz, M. F. (2006). Desarrollo profesional docente. Granada: Grupo Editorial Universitário.

Dall'Alba, G., \& Sandberg, J. (2006). Unveiling professional development: A critical review of stage models. Review of Educational Research, 76(3), 383-412. Disponível em: http://rer. sagepub.com/content/76/3/383

Darling-Hammond, L. (2006). Constructing 21 st-century teacher education. Journal of 
Teacher Education Policy Analysis Archives, 57(3), 300-314.

Day, C. (2001). Desenvolvimento profissional de professores: Os desafios da aprendizagem permanente. Porto: Porto Editora.

DeWert, M. H., Babinski, L. M., \& Jones, B. D. (2003). Safe passages: Providing online support to beginning teachers. Journal of Teacher Education, 54(4), 311-320.

Dubar, C. (1995). La socialisation : Construction des identités sociales et professionnelles. Paris : Armand Colin.

Earley, P., \& Bubb, S. (2013). A day in the life of new headteachers: Learning from observation. Educational Management Administration \& Leadership, 41(6), 782-799.

Elmore, R. F. (2000). Building a new structure for school leadership. Washington, DC: The Albert Shanker Institute.

Elmore, R. F. (2006). Leadership as the practice of improvement. Comunicação apresentada na conferência International perspectives on school leadership for systemic improvement, 6 jul., Londres. Disponível em: http://www.oecd. org/edu/school/37133264.pdf

Esteves, M., \& Rodrigues, A. (Orgs.). (1993). Análise de necessidades na formação de professores. Porto: Porto Editora.

Estrela, A., \& Estrela, M. T. (2006). A formação contínua de professores numa encruzilhada. In R. Bizarro \& F. Braga (Eds.), Formação de professores de línguas estrangeiras: Reflexões, estudos e experiências (pp. 73-80). Porto: Porto Editora.

Estrela, M. T., Esteves, M., \& Rodrigues, A. (2002). Síntese da investigação sobre formação inicial de professores em Portugal (1990-2000). Porto: Porto Editora.

Eurydice. (2002). The teaching profession in Europe: Profile trends and concerns. Report 1: Initial training and transition to working life. Brussels: European Commission.

Feiman-Nemser, S. (2008). How teachers learn to teach. In M. Cochran-Smith, S. Feiman-Nemser, \& D. J. Mcintyre (Eds.),
Handbook of research on teacher education. Enduring questions in changing contexts (pp. 697-705). New York: Routledge.

Fessler, R. (1995). Dynamics of teachers career stages. In T. R. Guskey, \& M. Huberman (Eds.), Professional development in education: New paradigms and practices (pp. 171-192). New York: Teachers College Press.

Fialho, I., Saragoça, J., Silvestre, M. J., Correia, A. P., \& Gomes, S. (2014). Relatórios internacionais e nacionais. Um olhar sobre a avaliação externa de escolas. In J. Pacheco (Ed.), Avaliação externa de escolas: Quadro teórico-conceptual (pp. 147-180). Porto: Porto Editora.

Flores, I., Mendes, R., \& Velosas, P. (2013). $\mathrm{O}$ que se passa que os alunos não passam? In Conselho Nacional de Educação, Estado da educação 2013 (pp. 374-391). Lisboa: Conselho Nacional de Educação.

Flores, M. A. (1999). (Des)ilusões e paradoxos: A entrada na carreira na perspectiva dos professores neófitos. Revista Portuguesa de Educação, 12(1), 171-204.

Flores, M. A. (2004). Os professores em início de carreira e o seu processo de mudança: Influências e percursos. Revista Educação, 12(2), 173-190.

Flores, M. A., \& Ferreira, F. I. (2009). The induction and mentoring of new teachers in Portugal: Contradictions, needs and opportunities. Research in Comparative and International Education, 4(1), 63-73.

Fullan, M. (1990). Staff development, innovation and institutional change. In B. Joyce (Ed.), Changing school culture through staff development (pp. 3-25). USA: ASCD.

Ghiglione, R., \& Matalon, B. (1997). O inquérito: Teoria e prática ( $3^{\mathrm{a}}$ ed.). Oeiras: Celta.

Gonçalves, J. A. (1992). A carreira das professoras do ensino primário. In A. Nóvoa (Ed.), Vidas de professores (pp. 141-170). Porto: Porto Editora.

Gonçalves, J. A. (2009). Desenvolvimento 
profissional e carreira docente - Fases da carreira, currículo e supervisão. Sísifo. Revista de Ciências da Educação, 8, 23-36.

Greenfield, W. (1995). Toward a theory of educational administration: The centrality of leadership. Educational Administration Quarterly, 31(1), 61-85.

Grimsath, G., Nordvik, G., \& Bergsvik, E. (2008). The newly qualified teacher: A leader and a professional? A Norwegian study. Journal of In-Service Education, 34(2), 219-236.

Hadar, L., \& Brody, D. (2010). From isolation to symphonic harmony: Building a professional development community among teacher educators. Teaching and Teacher Education, 26, 1641-1651.

Hargreaves, A. (1998). Os professores em tempos de mudança: O trabalho e a cultura dos professores na idade pós-moderna. Lisboa: McGraw Hill.

Harland, T., \& Staniforth, D. (2006). Contrasting views of induction: The experiences of new academic staff and their heads of department. Active Learning in Higher Education, 7, 185-196.

Horng, E. L., Klasik, D., \& Loeb, S. (2009). Principal time-use and school effectiveness. School leadership research report No. 09-3. Stanford, CA: Stanford University, Institute for Research on Education Policy \& Practice.

Horng, E., \& Loeb, S. (2010). New thinking about instructional leadership. Phi Delta Kappan, 92(3), 66-69.

Huberman, M. (1992). O ciclo de vida profissional dos professores. In A. Nóvoa (Ed.), Vidas de professores (pp. 58-69). Porto: Porto Editora.

Kelchtermans, G. (2009). O comprometimento profissional para além do contrato: Autocompreensão, vulnerabilidade e reflexão dos professores. In M. A. Flores, \& A. M. Simão (Eds.), Aprendizagem e desenvolvimento profissional dos professores: Contextos $e$ perspectivas (pp. 61-98). Mangualde: Edições Pedago.
Kessels, C. (2010). The influence of induction programs on beginning teachers' well-being and professional development. Leiden: ICLON, Leiden University Graduate School of Teaching.

Leithwood, K. A. (1990). The principal's role in teacher development. In B. Joyce (Ed.), Changing school culture through staff development (pp. 71-90). USA: ASCD.

Leithwood, K. A., \& Riehl, C. (2003). What we know about successful school leadership. Philadelphia, PA: Laboratory for Student Success, Temple University.

Leithwood, K.A., Day, C., Sammons, P., Harris, A., \& Hopkins, D. (2006). Seven strong claims about successful school leadership. Nottingham: National College of School Leadership.

Lima, L. (2014). A gestão democrática das escolas: Do autogoverno à ascensão de uma pós-democracia gestionária?. Educação \& Sociedade, 35(129), 1067-1083.

Mahon, R., \& McBride, S. (2008). Introduction. In R. Mahon, \& S. McBride (Eds.), The OECD and transnational governance (pp. 3-22). Vancouver: UBC Press.

Marable, M., \& Raimondi, S. L. (2007). Teachers' perceptions of what was most (and least) supportive during their first year of teaching. Mentoring and Tutoring, 15(1), 2537.

Marcelo, C. (1991) Aprender a enseñar: Un estudio sobre el proceso de socialización de profesores principiantes. Madrid: CIDE.

Marcelo, C. (2009). Desenvolvimento profissional docente: Passado e futuro. Sísifo. Revista de Ciências da Educação, 8, 7-22.

Marôco, J. (2018). Análise estatística com o SPSS Statistics ( $7^{\text {a }}$ Ed.). Pêro Pinheiro: ReportNumber.

Ministério da Educação. (2007). Política de formação de professores em Portugal. Lisboa: ME/DGRHE.

Ministério da Educação e Ciência. (2017). Educação em números - Portugal 2016. Lisboa: 


\section{MEC/DGEEC.}

Molner Kelley, L. (2004). Why induction matters. Journal of Teacher Education, 55(5), 438-448.

Morais, F., \& Medeiros, T. (2007). Desenvolvimento profissional do professor - A chave do problema. Ponta Delgada: Universidade dos Açores \& DRCT.

Murillo, F. J. (2003). El movimiento teóricopráctico de mejora de la escuela. Algunas lecciones aprendidas para transformar los centros docentes. Revista Iberoamericana sobre Calidad, Eficacia y Cambio en Educación, 1(2), 1-22. Disponível em: https://revistas.uam.es/ index.php/reice/article/view/5352

Northouse, P. G. (2010). Leadership: Theory and practice ( $5^{\mathrm{a}}$ ed.). Thousand Oaks, CA: Sage.

O'Malley, M. P., Long, T. A., \& King, J. (2015). "What do you do all day?": Navigating the challenges of school leadership as an early career principal. Journal of Cases in Educational Leadership, 18(2), 107-121.

OECD. (2005). Attracting, developing and retaining effective teachers. Final report: Teachers matter. Paris: OECD Publications.

Oliveira-Formosinho, J. (2000). O desenvolvimento profissional de educadores de infância principiantes: Relato de uma investigação. Infância e Educação: Investigação e Práticas, 2, 109-124.

Rivkin, S., Hanushek, E. A., \& Kain, J. F. (2005). Variable definitions, data, and programs for "Teachers, students, and academic achievement". Econometrica Supplementary Material, 73(2), 417-458.

Roldão, M. C., Reis, P., \& Costa, N. (2012). Da incoerência burocrática à eficácia de um dispositivo de supervisão/formação. Estudo do desenvolvimento profissional numa situação de indução. Ensaio, 20(76), 435-458.

Santiago, P., Roseveare, D., Van Amelsvoort, G., Manzi, J., \& Matthews, P. (2009). Teacher evaluation in Portugal. Paris: OECD Publishing. Serrazina, L., \& Oliveira, I. (2002). Novos professores: Primeiros anos de profissão. Quadrante, 11(2), 55-73.

Shulman L. (2004). The wisdom of practice. Essays on teaching, learning, and learning to teach. San Francisco: Jossey- Bass.

Sikes, P. (1992). The life cycle of the teacher. In S. Ball, \& I. Goodson (Eds.), Teachers' lives and careers (pp. 27-60). Philadelphia: Falmer Press.

Stansbury, K. (2001). What new teachers need. Leadership, 30(3), 18-21.

Steffy, B. E., Wolf, M. P., Pash, S. H., \& Enz, B. J. (2000). The life cycle of the teacher career. California: Corwin Press.

Sunde, E., \& Ulvik, M. (2014). School leaders' views on mentoring and newly qualified teachers' needs. Education Inquiry, 5(2), 285299.

Torres, L. L. (2013). Liderança singular na escola plural: As culturas da escola perante o processo de avaliação externa. Revista Lusófona de Educação, 23, 51-76.

Uwamariya, A., \& Mukamurera, J. (2005). Le concept de "développement professionnel" en enseignement : Approches théoriques. Revue des Sciences de L'Éducation, 31(1), 133-155.

Veenman, S. (1984). Perceived problems of beginning teachers. Review of Educational Research, 54, 143-178.

Villegas-Reimers, E. (2003). Teacher professional development: An international review of the literature. Paris: UNESCO.

Waters, T., \& Grubb, S. (2004). Leading schools: Distinguishing the essential from the important. Denver, CO: Mid-continent Research for Education and Learning.

Whithaker, P. (2000). Gerir a mudança na escola. Porto: Asa.

Wischkaemper, K. A. (2005). A comparative case study of the principal's role in new teacher induction (Tese de doutoramento). Graduate Faculty of Texas Tech University, Texas.

Yukl, G. A. (2002). Leadership in organizations. New York: Prentice Hall.

Zabalza, M. (2007). Competencias docentes 
del profesorado universitario: Calidad $y$ desarrollo profesional. Madrid: Narcea.

Zeichner, K., \& Gore, J. (1990). Teacher socialization. In W. Houston, \& W. Robert (Eds.), Handbook of research on teacher education (pp. 329-348). New York: MacMillan.

\section{LEG ISLAÇ Ã O CONSULTA DA}

Portugal. (1986). Lei de Bases do Sistema Educativo. Lei n. ${ }^{\circ} 46 / 86$, de 14 de outubro. Diário da República n. ${ }^{\circ}$ 237/1986, Série I.

Portugal. (1990). Decreto-lei n. ${ }^{\circ}$ 139-A/90, de 28 de abril. Estatuto da Carreira dos Educadores de Infância e dos Professores dos Ensinos Básicos e Secundário.

Portugal. (2008). Decreto-lei n. ${ }^{\circ} 75 / 2008$, de 22 de abril. Diário da República n. ${ }^{\circ}$ 79/2008, Série I. Aprova o regime de autonomia, administração e gestão dos estabelecimentos públicos da educação pré-escolar e dos ensinos básico e secundário

Portugal. (2013a). Lei n. ${ }^{\circ} 80 / 2013$, de 28 de novembro. Diário da República n. ${ }^{\circ}$ 231/2013, Série I. Estabelece o regime jurídico da requalificação de trabalhadores em funções públicas.

Portugal. (2013b). Conselho Nacional de Educação. Recomendação no 4/2013, de 17 de maio. Diário da República n. 95/2013, Série II. Recomendação do Conselho Nacional de Educação sobre formação contínua de educadores de infância e professores dos ensinos básico e secundário.

Portugal. (2015). Despacho n. ${ }^{\circ} 9488 / 2015$, de 20 de agosto. Diário da República n. ${ }^{\circ}$ 162/2015, Série II. Período probatório de docentes - 2015.
ADDRESSING TEACHER'S INDUCTION: WHAT ARE THE IMPLICATIONS FOR PORTUGUESE SCHOOL PRINCIPALS?

\section{Abstract}

The article focuses on the role played by the Portuguese school principals in promoting the integration and professional development of beginning teachers in their organisations. Developed within the framework of the European Project The Outstanding New Teacher Programme (ONTP), the article presents the results of a quantitative study, by using a questionnaire technique, applied in electronic format. We tried to understand what needs school principals have in order to welcome and foster the professional growth of teachers at the beginning of their activity. Data was analysed, primarily, through descriptive analyses on each item and the dimensions of analysis of teachers' needs (promotion of professional and organizational development, pedagogical leadership and work organization); secondly, through inferential analyses according to the characteristics of the subjects, seen as capable of explaining some variability in the responses. The results allow us to infer valuing observation and reflection among peers as strategies for professional and organizational development. It is evident the need for support to exercise pedagogical leadership by school principals, particularly considering that the higher their level of academic qualification, the stronger those needs are stated.

KEYWORDs: School principals; Pedagogical leadership; Professional development; Beginning teachers 


\section{AgIR SUR L'INDUCTION DES ENSEIGNANTS : QUELLES SONT LES IMPLICATIONS POUR LES CHEFS D'ETABLISSEMENT PORTUGAIS?}

\section{RÉSUMÉ}

Larticle se concentre sur le rôle des chefs détablissement portugais dans la promotion de l'intégration et du développement professionnel des enseignants débutants dans leurs organisations. Développé dans le cadre du Projet Européen The Outstanding New Teacher Programme (ONTP), l'article présente les résultats de l'étude quantitative, en utilisant la technique $\mathrm{du}$ questionnaire, appliquée en format électronique, où l'on a cherché à percevoir quels sont les besoins ressentis par les directeurs pour accueillir et favoriser la croissance professionnelle des enseignants au début de leur activité. Les données collectées ont été analysées selon deux procédures: dans la première phase, des analyses descriptives ont été effectuées sur chaque élément de l'instrument et sur les dimensions de l'analyse des besoins des enseignants (dans le but de promouvoir le développement professionnel et organisationnel, la gestion pédagogique et l'organisation du travail); et, deuxièmement, des analyses inférentielles ont été effectuées en fonction des caractéristiques des chefs d'établissement, considérés comme des variables capables d'expliquer une certaine variabilité dans les réponses. Les résultats nous permettent de déduire une évaluation de lobservation et de la réflexion chez les enseignants en tant que stratégies de développement professionnel et organisationnel. Le besoin déclaré de soutien pour exercer la dimension de leadership pédagogique en tant que directeurs est assez évident, et il devient d'autant plus fort que le niveau de qualification académique est élevé.

Mots-CLÉs : Chef d'établissement ; Leadership pédagogique ; Développement professionnel ; Enseignants débutants

\footnotetext{
${ }^{\text {I }}$ Unidade de Investigação e Desenvolvimento em Educação Formação (UIDEF), Instituto de Educação, Universidade de Lisboa, Portugal. Orcid: 0000-0003-3108-4289

II Unidade de Investigação e Desenvolvimento em Educação Formação (UIDEF), Instituto de Educação, Universidade de Lisboa, Portugal. Orcid: 0000-0003-1525-3433

III Unidade de Investigação e Desenvolvimento em Educação Formação (UIDEF), Instituto de Educação, Universidade de Lisboa, Portugal. Orcid: 0000-0002-1814-1042

IV Instituto de Educação, Universidade de Lisboa, Portugal. Orcid: 0000-0001-7150-145X
} 